\title{
Bracing after percutaneous vertebroplasty for thoracolumbar osteoporotic vertebral compression fractures was not effective
}

This article was published in the following Dove Medical Press journal:

Clinical Interventions in Aging

\author{
Jianan Zhang \\ Yong Fan \\ Xin $\mathrm{He}$ \\ Jinpeng $\mathrm{Du}$ \\ Dingjun Hao
}

Department of Spine Surgery,

Hong Hui Hospital, Xi'an Jiaotong

University, Xi'an 7I0054, Shaan'xi

Province, China
Correspondence: Dingjun Hao

Department of Spine Surgery, Hong Hui Hospital, Xi'an Jiaotong University,

No 76 Nanguo Road, Nanshao Gate,

Xi'an 7I 0054, Shaan'xi Province, China

Tel +86 I3909297839

Fax +86 2987894724

Email haodjspine@।26.com
Objective: The objective of this study was to evaluate the effectiveness of bracing after percutaneous vertebroplasty (PVP) for thoracolumbar osteoporotic vertebral compression fractures (OVCF).

Methods: This is a retrospective study where we recruited 138 patients with single-level thoracolumbar OVCF who underwent PVP from January 2018 to March 2018 without bracing after PVP (Group A). The visual analog score (VAS) and vertebral body compression ratio (VCR) were recorded at preoperation, on the second day, at 2 weeks, 1 month, and 6 months after operation. Oswestry Disability Index (ODI) was recorded at preoperation, 2 weeks, 1 month, and 6 months after operation. Propensity score matching identified 138 historical patients (Group B) as controls, who used rigid brace for 3 weeks after the surgery, from January 2017 to December 2017 using six independent variables (preoperation): age, sex, VAS, ODI, bone mineral density, and body mass index. The indicators and complications were compared between the two groups. Results: Compared with preoperation, VAS and VCR were significantly improved $(P<0.05)$ in both groups on the second day after operation. At 2 weeks, 1 month, and 6 months after PVP operation, ODI, VAS, and VCR were all significantly improved than at preoperation $(P<0.05)$. There were no statistical differences between the two groups in VAS and VCR on the second day, at 1 month and 6 months after PVP $(P>0.05)$. There were no significant differences between Groups A and B in ODI at 2 weeks and 6 months after operation $(P>0.05)$ but ODI for Group B at 1 month after operation was significantly higher than Group A $(P<0.05)$. Eleven cases in Group A and 13 cases in Group B had poor pain relief on the second day after operation, and there were no significant differences in VAS and ODI between the two subgroups at 2 weeks, 1 month, and 6 months after PVP. There were no significant differences in the collapse and refracture rates between the two groups.

Conclusion: In summary, in terms of quality of life and complications after operation, postoperative bracing did not result in improved outcomes. Presence or absence of bracing did not relieve postoperative residual pain. In contrast, bracing for 3 weeks after operation reduced the quality of life in the short term.

Keywords: percutaneous vertebroplasty, osteoporotic vertebral compression fractures, brace

\section{Introduction}

Osteoporotic vertebral compression fractures (OVCF) often lead to severe back pain, sleep loss, kyphosis, decreased quality of life, and increased risk of age-adjusted mortality. ${ }^{1-3}$ In addition to anti-osteoporotic treatment, conservative treatments include analgesic medications, passive physical therapies, bed rest, or use of brace. ${ }^{4}$ Percutaneous vertebroplasty (PVP) is an effective treatment for patients with surgical 
indications, ${ }^{5}$ which can significantly relieve the pain, reduce the bed rest time, and prevent deformity due to collapse of the vertebral body. ${ }^{6}$ Previous studies mostly focused on surgical method, non-surgical method, and surgical complications. Some studies reported collapse of the same vertebra at an incidence rate of $3.21 \%-10 \%$ after PVP or percutaneous kyphoplasty (PKP), and it occurred primarily within the first few months after operation. ${ }^{7,8}$ Some doctors suggested that patients should wear a spinal brace after PVP to prevent vertebral collapse, osteonecrosis, and back pain..$^{9-11}$ To our knowledge, no reports have confirmed whether bracing after cement augmentation could offer additional clinical benefit. In this study, we evaluated the effectiveness of bracing after PVP.

\section{Materials and methods}

\section{Study patients}

The study patients were admitted into our hospital between January 2017 and March 2018. Inclusion criteria included: 1) single-level OVCF, 2) treatment with PVP via unilateral portals, 3 ) bone mineral density (BMD) of 2.5 or lower, and 4) use of anti-osteoporotic medications during the follow-up period. Exclusion criteria included: 1) history of malignancy, infection, or tumor; 2) history of trauma; 3) previous PVP or PKP for OVCF; 4) bone cement did not touch both upper and lower end plates; 5) OVCF with intervertebral cleft, and 6) patients who used steroids.

We confirmed that all methods were carried out in accordance with relevant guidelines and regulations of ethics committee of Xi'an Hong Hui Hospital. We confirmed that all experimental protocols were approved by the ethics committee of Xi'an Hong Hui Hospital and all procedures performed in this study, involving human participants, were in accordance with the ethical standards of the institutional research committee and with the 1964 Helsinki Declaration and its later amendments or comparable ethical standards. Written informed consent was obtained from all patients for this study.

\section{Surgical procedure}

The PVP was performed using specialized instruments (Vertebroplasty system; Kinetic, Shanghai, China) to inject polymethylmethacrylate (PMMA) cement (Mendec Spine Resin; Tecres S.P.A, Andrea Doria, Italy) with unilateral transverse process-pedicle under local anesthesia. ${ }^{12}$ The operations were performed by three different surgeons independently.

\section{Postoperative care}

Out-of-bed activity was allowed 6 hours after the operation. The patients were discharged 3 days after surgery. Some of the previous studies suggested wearing a spinal brace for 4-8 weeks after PVP, and there was no exact time for bracing after PVP in any treatment guide. ${ }^{9-11}$ Based on our clinical experience and previous studies, we thought that bracing for a long time after PVP may not provide benefit anymore, so we only choose 3 weeks as the bracing period after operation. A rigid brace was applied after the operation for 3 weeks to all patients admitted before December 2017 (Figure 1). From January 2018 onward, brace application after PVP was not recommended. Anti-osteoporotic drugs (including calcium carbonate, calcitriol, and alendronate) were prescribed for at least 6 months after the operation. All patients were instructed to perform back and abdominal muscle exercises for 4 weeks after discharge.

\section{Radiographic and clinical evaluation}

Demographic and operative data were recorded and compared between the two groups. The visual analog score (VAS) and vertebral body compression ratio (VCR) were recorded and compared at preoperation, on second day, at 2 weeks, 1 month, and 6 months after operation. Adjusted Oswestry Disability Index (ODI; excluding the section of sex life) was also recorded and compared for the same time points. Postoperative residual pain was defined as pain relief (VAS) of $<50 \%$ on the second day after operation. In order to evaluate the efficacy of bracing after PVP for patients with postoperative residual pain, an additional separate analysis was performed for patients with postoperative residual pain. Collapse rate of operated vertebral body was compared between the two groups at 6 months after operation. Any relevant postoperative complications were recorded. The VCR was defined as the ratio between the anterior and posterior vertebral body height of the injured level. ${ }^{13}$ Considering the

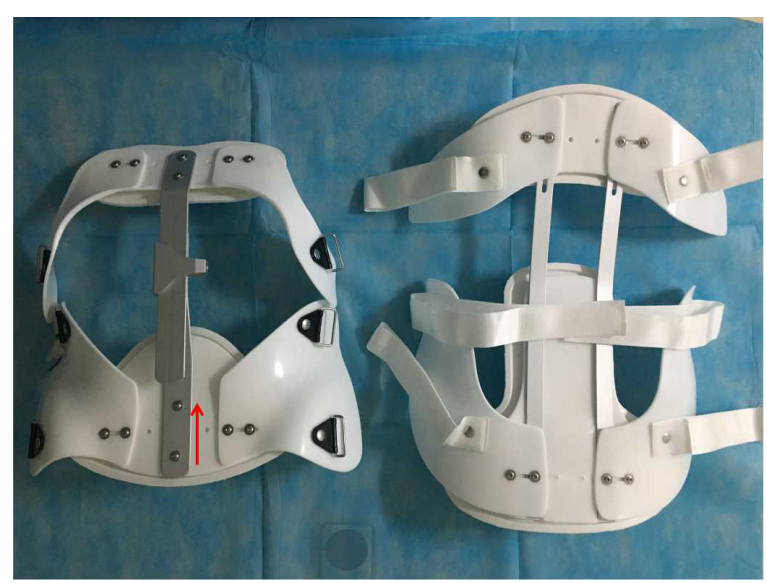

Figure I The spinal brace used in this study (thoracolumbosacral orthosis), which can be adjusted according to the height and weight of the patient (red arrow indicates the front side and the head side). 
measurement error, collapse of the operated vertebral body was defined as the difference between final anterior body height and the postoperative anterior body height exceeding $2.0 \mathrm{~mm} .{ }^{9} \mathrm{VCR}$ and collapse rate were measured independently by two of the co-authors (Dr Fan and Dr He), and the results were then averaged. During follow-up, refracture was recorded and defined as a patient having severe back pain during the follow-up with magnetic resonance imaging confirmation of the new spinal fracture.

\section{Statistical analysis}

Age, sex, VAS, ODI, BMD, and body mass index were set as independent variables for pairing by propensity matching with a 1:1 ratio. Normal distributions of the correlation data were confirmed by Kolmogorov-Smirnov test, and homogeneity of variance was tested by Levene test. Paired $t$-test and independent samples $t$-test were performed. Chi-squared test was performed to compare the fracture level, collapse rate, and gender. The statistical analyses were conducted using SPSS version 22.0 (SPSS Inc., Chicago, IL, USA). $P<0.05$ was considered statistically significant, and all $P$-values presented were two-tailed.

\section{Results}

A total of 1,256 patients with single-level OVCF underwent PVP or PKP from January 2017 to December 2017. Among these, 151 patients underwent a bilateral PVP or PKP, 78 patients had long-term steroid therapy, 129 patients were lost to follow-up, 120 patients had previous PVP or PKP, and three patients were diagnosed with multiple myeloma after operation. Twenty-three patients refused to wear the brace after surgery, and 16 patients prolonged bracing because of low back discomfort when removed. Finally, there were 736 patients in the historical control group; of them, 138 patients were paired by propensity score into Group B. Moreover, there were no statistical differences between the baseline values of the two groups (Table 1).

Compared with preoperative values, VAS and VCR were significantly improved $(P<0.05)$ in both groups on the second day after operation. At 2 weeks, 1 month, and 6 months after PVP operation, the ODI, VAS, and VCR were all significantly improved than the values at preoperation $(P<0.05)$. The ODI at 1 month postoperation in Group B was significantly higher than in Group A $(P<0.05$, Table 2$)$. There were no significant differences in ODI at 2 weeks and 6 months after operation between the two groups $(P>0.05)$. There were no statistical differences in VAS or VCR between the two groups at 2 weeks, 1 month, and 6 months after PVP $(P>0.05)$.
Table I The relevant data of patients

\begin{tabular}{|c|c|c|c|}
\hline Characteristics & Group A & Group B & $P$-value \\
\hline Case & 138 & 138 & \\
\hline Age (years) & $66.4 \pm 4.9$ & $66.8 \pm 5.0$ & 0.503 \\
\hline Sex (male/female) & $52 / 86$ & $49 / 89$ & 0.708 \\
\hline $\mathrm{BMD} \mathrm{g} / \mathrm{cm}^{3}$ & $-3.12 \pm 0.43$ & $-3.08 \pm 0.38$ & 0.414 \\
\hline The volume of bone cement & $5.16 \pm 1.33$ & $5.47 \pm 1.52$ & 0.073 \\
\hline $\mathrm{BMI} \mathrm{kg} / \mathrm{m}^{2}$ & $23.16 \pm 3.14$ & $22.74 \pm 3.92$ & 0.323 \\
\hline \multicolumn{4}{|l|}{ Level } \\
\hline TIO & 14 & II & \multirow[t]{5}{*}{0.120} \\
\hline TII & 29 & 17 & \\
\hline $\mathrm{T} 12$ & 38 & 45 & \\
\hline LI & 35 & 38 & \\
\hline L2 & 22 & 27 & \\
\hline \multicolumn{4}{|l|}{ Preoperation } \\
\hline ODI & $65.82 \pm 5.64$ & $66.21 \pm 6.18$ & 0.584 \\
\hline VAS & $6.36 \pm 0.60$ & $6.45 \pm 0.93$ & 0.340 \\
\hline VCR & $0.65 \pm 0.09$ & $0.64 \pm 0.05$ & 0.255 \\
\hline
\end{tabular}

Note: Data shown as mean \pm SD or number.

Abbreviations: BMD, bone mineral density (lumbar and left hip); BMI, body mass index; ODI, Oswestry Disability Index; VAS, visual analog score; VCR, vertebral body compression ratio.

There were no severe operative complications, such as postoperative neurologic deficit or pulmonary embolism due to leakage of bone cement. We did not assess the respiratory function in our study, but four patients in Group B complained about the brace for respiratory reasons. Eleven cases in

Table 2 The clinical and radiological results

\begin{tabular}{|l|l|l|l|}
\hline Characteristics & Group A & Group B & P-value \\
\hline \multicolumn{5}{|l|}{ The second day after operation } \\
\hline VAS & $2.27 \pm 1.53$ & $2.49 \pm 1.57$ & 0.240 \\
\hline VCR & $0.73 \pm 0.15$ & $0.75 \pm 0.13$ & 0.238 \\
\hline 2 weeks after operation & $22.56 \pm 6.58$ & $23.49 \pm 6.36$ & 0.234 \\
\hline ODI & $2.12 \pm 1.31$ & $2.26 \pm 1.39$ & 0.390 \\
\hline VAS & $0.68 \pm 0.17$ & $0.71 \pm 0.14$ & 0.111 \\
\hline VCR & $21.24 \pm 5.7$ & $22.97 \pm 6.85$ & $0.023 *$ \\
\hline I month after operation & $2.03 \pm 1.06$ & $2.12 \pm 1.27$ & 0.523 \\
\hline ODI & $0.67 \pm 0.12$ & $0.66 \pm 0.14$ & 0.525 \\
\hline VAS & $15.28 \pm 2.79$ & $15.94 \pm 3.57$ & 0.088 \\
\hline VCR & $1.56 \pm 0.72$ & $1.71 \pm 0.79$ & 0.100 \\
\hline 6 months after operation & $0.66 \pm 0.13$ & $0.64 \pm 0.15$ & 0.238 \\
\hline ODI & $9 / 129$ & $13 / 125$ & 0.374 \\
\hline VAS & $22 / 116$ & $27 / 111$ & 0.431 \\
\hline VCR &
\end{tabular}

Notes: Data shown as mean \pm SD or number. *Statistical significance.

Abbreviations: ODI, Oswestry Disability Index; VAS, visual analog score; VCR, vertebral body compression ratio. 
Table 3 Clinical outcomes of patients with postoperative residual pain

\begin{tabular}{|l|l|l|l|}
\hline Characteristics & Group A $(\mathbf{n}=\mathbf{I} \mathbf{I})$ & Group B $(\mathbf{n}=\mathbf{I} \mathbf{3})$ & P-value \\
\hline \multicolumn{4}{|l|}{ The second day after operation } \\
\hline VAS & $4.3 \pm 1.00$ & $4.00 \pm 0.91$ & 0.454 \\
\hline VCR & $0.71 \pm 0.11$ & $0.72 \pm 0.09$ & 0.812 \\
\hline 2 weeks after operation & $31.24 \pm 5.60$ & $28.22 \pm 5.24$ & 0.190 \\
\hline ODI & $3.22 \pm 0.98$ & $2.92 \pm 0.76$ & 0.416 \\
\hline VAS & $24.07 \pm 4.93$ & $25.34 \pm 6.28$ & 0.585 \\
\hline I month after operation & $2.54 \pm 0.66$ & 0.448 \\
\hline ODI & $2.78 \pm 0.83$ & $17.21 \pm 2.89$ & 0.088 \\
\hline VAS & $16.79 \pm 2.65$ & $1.85 \pm 0.69$ & 0.767 \\
\hline 6 months after operation & $1.78 \pm 0.44$ &
\end{tabular}

Note: Data shown as mean \pm SD.

Abbreviations: ODI, Oswestry Disability Index; VAS, visual analog score; VCR, vertebral body compression ratio.

Group A and 13 cases in Group B complained about noticeable back pain on the second day after operation. These 24 patients were given celecoxib to relieve pain, and the pain was significantly reduced at the last follow-up. There were no significant differences in VAS and ODI between these two subgroups at 2 weeks (Table 3 ). There were no significant differences in collapse rate and refracture rate between the two groups, as shown in Figure 2 and Table 2.

\section{Discussion}

Since PVP with PMMA augmentation was reported in 1987 , PVP has been widely advocated as an effective treatment technique for OVCF. ${ }^{14}$ In this study, VAS, ODI, and VCR in both groups achieved significantly satisfactory results compared to preoperative values. This is in agreement with previous literature. ${ }^{6,15}$ Studies in recent years were mainly focused on leakage of bone cement, adjacent vertebra refracture, and injury of vessel and/or nerve. However, after reviewing some related literature, there were varied descriptions on the use of a brace in early stage of recovery following PVP or PKP. Even though the literature recommended the use of a brace after cement augmentation, the bracing period varied from 4 to 8 weeks..$^{9-11}$ It is still unclear whether bracing after PVP could prevent postoperative complications and improve quality of life. According to the previous studies, risk factors, such as BMD, intervertebral cleft, volume of bone cement, and so on, were associated with collapse of vertebral body, osteonecrosis after PVP, and postoperative satisfaction. ${ }^{16,17}$ Certainly, OVCF with intervertebral cleft is an important indication for surgical treatment, and because the sample size of the patients without bracing after PVP with intervertebral cleft was small, it was not included in the study, and we will discuss this topic in our future research. In order to reduce the bias of this retrospective study, the control group (Group B) based on propensity score matching was included, and there were no statistical differences in the baseline parameters between the two groups $(P>0.05)$.

Bracing has been widely used in the management of thoracolumbar fractures treated with or without surgical stabilization. In theory, a spinal brace could relieve the pain, provide stability to the fracture site, reduce the bed rest time, and prevent further kyphotic collapse. ${ }^{18}$ The results of this study showed that there were no significant differences in the VCR between the two groups at 2 weeks, 1 month, and 6 months after operation, and there were no significant differences in collapse rate. Moreover, we observed that most patients will lose a little vertebral height since self-ambulation was permitted, but this phenomenon
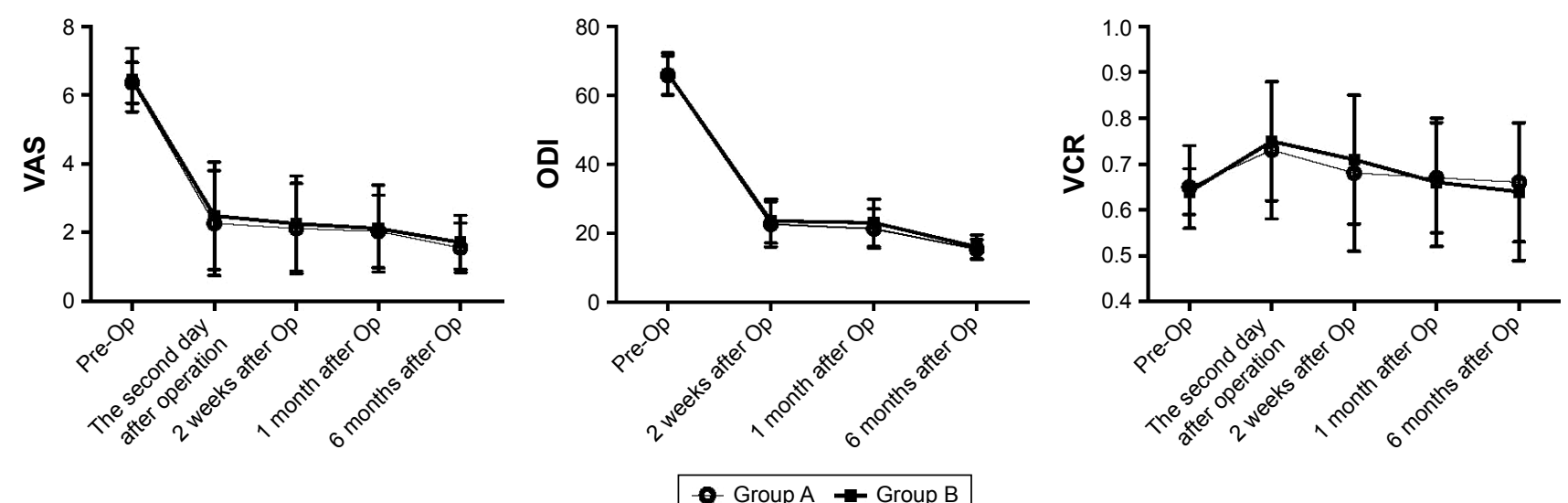

Figure 2 Comparison of ODI, VAS, and VCR among the two groups.

Abbreviations: ODI, Oswestry Disability Index; VAS, visual analog score; VCR, vertebral body compression ratio; Op, operation. 
did not cause discomfort. Even though bracing was proven to be an effective method for treating certain thoracolumbar fractures in many cases, ${ }^{19} \mathrm{OVCF}$ are inherently stable fractures, especially after cement augmentation, where local stability has been achieved. ${ }^{20}$ The brace may restrict spinal motion in some diseases, but this did not mean bracing could provide additional benefits for relatively stable patients, as confirmed in previous studies which focused on cervical and lumbar spinal surgery. ${ }^{21,22}$ Even for the patients with adolescent idiopathic scoliosis, postoperative bracing was unnecessary after internal fixation. ${ }^{23}$ Although the use of brace after PVP or PKP is reported in some studies, there was no evidence of benefits from additional external immobilization. A prospective randomized controlled study from Kim et al showed that for conservative treatment of OVCF, progression of anterior body compression, improvement of back pain, and ODI without a brace were not inferior to those with soft or rigid braces. ${ }^{13}$ Bailey et al have also reported results consistent with this finding. ${ }^{24}$ In our study, there were no statistical differences in VAS between the two groups on the second day, at 2 weeks, 1 month, and 6 months after PVP $(P>0.05)$. There were no significant differences in ODI between Groups A and B at 2 weeks and 6 months after operation $(P>0.05)$. Based on the results of a review study, postoperative bracing did not improve outcomes after cervical and lumbar surgery. ${ }^{25}$ The review study found that internal fixation in spinal surgery has allowed for rigid internal stabilization of the cervical and lumbar surgery, and decreased the requirement for external immobilization. As expected, since the patients did not benefit from additional external immobilization, the back pain due to OVCF was likely the main factor influencing the daily life and ODI, and the operation relieved the pain almost immediately.

ODI at 1 month after operation in Group B was significantly higher than in Group A. Group B achieved higher scores, especially for sitting, standing, and traveling. It is likely that the core muscle atrophy in Group B due to extended bracing would cause back pain and discomfort, hence could reduce the quality of life. This study also highlighted that bracing is associated with some disadvantages and should be more vigilantly monitored for the elderly, since bracing could lead to muscular atrophy, impaired respiration, skin irritation, and additional costs. ${ }^{13,18}$ However, at 6 months after operation, there was no significant difference in ODI between the two groups, and this may be related to core muscle strengthening by back and abdominal muscle exercises.
The results showed that even for patients who still had obvious pain after operation, there were no significant differences in VAS and ODI between the two groups on the second day, at 2 weeks, 1 month, and 6 months after PVP $(P>0.05)$. Four patients in Group B did complain about the brace for respiratory reasons. Kato et al also found for OVCF that the use of brace can negatively affect the respiratory function of patients with spinal pathologies. ${ }^{26}$ The results also showed that there was no significant difference in refracture rate. To our knowledge, no reliable study to date has shown that bracing can reduce postoperative refracture rate. We initially hypothesized that short time bracing would not change the local biomechanical environment for a long time, and prolonged bracing might lead to trunk muscle atrophy, therefore may even increase refracture rate. If patients with refracture resisted conservative management or were not satisfied with pain relief after conservative treatment for 2-3 weeks, they were considered to have PVP/PKP, and at the same time, anti-osteoporotic drugs (including calcium carbonate, calcitriol, and alendronate) were prescribed for at least 6 months. Based on our results, the study indicated that bracing did not improve the quality of life and vertebral height; furthermore, the presence or absence of bracing did not relieve postoperative residual pain.

There are several limitations in our study. Firstly, since this is a retrospective study, a large randomized prospective controlled trial is needed to verify our results. Secondly, the follow-up to only 6 months after operation might not be sufficient for comparing the efficacy of bracing. Finally, the operations were performed by three different surgeons and inter-operator variability may have influenced the results.

\section{Conclusion}

In summary, in terms of quality of life and complications after operation, postoperative bracing did not result in improved outcomes. Presence or absence of bracing did not relieve postoperative residual pain. In contrast, bracing for 3 weeks after operation reduced the quality of life in the short term.

\section{Acknowledgment}

No funds were received in support of this work. No benefits in any form have been or will be received from a commercial party related directly or indirectly to the subject of this manuscript.

\section{Disclosure}

The authors report no conflicts of interests in this work. 


\section{References}

1. Burger H, van Daele PL, Grashuis K, et al. Vertebral deformities and functional impairment in men and women. J Bone Miner Res. 1997; 12(1):152-157.

2. Kado DM, Browner WS, Palermo L, Nevitt MC, Genant HK, Cummings SR. Vertebral fractures and mortality in older women: a prospective study. Study of osteoporotic Fractures Research Group. Arch Intern Med. 1999;159(11):1215-1220.

3. Silverman SL. The clinical consequences of vertebral compression fracture. Bone. 1992;13(Supp1 2):S27-S31.

4. Rzewuska M, Ferreira M, Mclachlan AJ, Machado GC, Maher CG. The efficacy of conservative treatment of osteoporotic compression fractures on acute pain relief: a systematic review with meta-analysis. Eur Spine J. 2015;24(4):702-714.

5. Xu Z, Hao D, He L, et al. An assessment system for evaluating the severity of thoracolumbar osteoporotic fracture and its clinical application: a retrospective study of 381 cases. Clin Neurol Neurosurg. 2015;139: $70-75$.

6. Klazen CA, Lohle PN, de Vries J, et al. Vertebroplasty versus conservative treatment in acute osteoporotic vertebral compression fractures (Vertos II): an open-label randomised trial. Lancet. 2010;376(9746): 1085-1092.

7. Heo DH, Chin DK, Yoon YS, Kuh SU. Recollapse of previous vertebral compression fracture after percutaneous vertebroplasty. Osteoporos Int. 2009;20(3):473-480.

8. Lavelle WF, Cheney R. Recurrent fracture after vertebral kyphoplasty. Spine J. 2006;6(5):488-493.

9. Kim YY, Rhyu KW. Recompression of vertebral body after balloon kyphoplasty for osteoporotic vertebral compression fracture. Eur Spine J. 2010;19(11):1907-1912.

10. Zhang L, Wang Q, Wang L, Shen J, Zhang Q, Sun C. Bone cement distribution in the vertebral body affects chances of recompression after percutaneous vertebroplasty treatment in elderly patients with osteoporotic vertebral compression fractures. Clin Interv Aging. 2017;12: $431-436$.

11. Savage JW, Schroeder GD, Anderson PA. Vertebroplasty and kyphoplasty for the treatment of osteoporotic vertebral compression fractures. J Am Acad Orthop Surg. 2014;22(10):653-664.

12. Yan L, He B, Guo H, Liu T, Hao D. The prospective self-controlled study of unilateral transverse process-pedicle and bilateral puncture techniques in percutaneous kyphoplasty. Osteoporos Int. 2016;27(5):1849-1855.

13. Kim HJ, Yi JM, Cho HG, et al. Comparative study of the treatment outcomes of osteoporotic compression fractures without neurologic injury using a rigid brace, a soft brace, and NO brace: a prospective randomized controlled non-inferiority trial. J Bone Joint Surg Am. 2014;96(23):1959-1966.
14. Galibert P, Deramond H, Rosat P, Le Gars D. Note préliminaire sur le traitement des angiomes vertébraux par vertébroplastie percutanée [Preliminary note on the treatment of vertebral angioma by percutaneous acrylic vertebroplasty]. Neurochirurgie. 1987;33(2):166-168. French.

15. Chin DK, Kim YS, Cho YE, Shin JJ. Efficacy of postural reduction in osteoporotic vertebral compression fractures followed by percutaneous vertebroplasty. Neurosurgery. 2006;58(4):695-700.

16. Hey HW, Tan JH, Tan CS, Tan HM, Lau PH, Hee HT. Subsequent vertebral fractures post cement augmentation of the thoracolumbar spine: does it correlate with level-specific bone mineral density scores? Spine. 2015;40(24):1903-1909.

17. Lin WC, Lee $\mathrm{YC}$, Lee $\mathrm{CH}$, et al. Refractures in cemented vertebrae after percutaneous vertebroplasty: a retrospective analysis. Eur Spine J. 2008;17(4):592-599.

18. Agabegi SS, Asghar FA, Herkowitz HN. Spinal Orthoses. Am Acad Orthop Surg. 2010;18(11):657-667.

19. Chang V, Holly LT. Bracing for thoracolumbar fractures. Neurosurg Focus. 2014;37(1):E3.

20. Denis F. The three column spine and its significance in the classification of acute thoracolumbar spinal injuries. Spine. 1983;8(8):817-831.

21. Yee AJ, Yoo JU, Marsolais EB, et al. Use of a postoperative lumbar corset after lumbar spinal arthrodesis for degenerative conditions of the spine. A prospective randomized trial. J Bone Joint Surg Am. 2008; 90(10):2062-2068.

22. Hida T, Sakai Y, Ito K, et al. Collar fixation is not mandatory after cervical laminoplasty: a randomized controlled trial. Spine. 2017;42(5): E253-E259.

23. Christodoulou AG, Prince HG, Webb JK, Burwell RG. Adolescent idiopathic thoracic scoliosis. A prospective trial with and without bracing during postoperative care. J Bone Joint Surg Br. 1987;69(1):13-16.

24. Bailey CS, Urquhart JC, Dvorak MF, et al. Orthosis versus no orthosis for the treatment of thoracolumbar burst fractures without neurologic injury: a multicenter prospective randomized equivalence trial. Spine J. 2014;14(11):2557-2564.

25. Zhu MP, Tetreault LA, Sorefan-Mangou F, Garwood P, Wilson JR. Efficacy, safety, and economics of bracing after spine surgery: a systematic review of the literature. Spine J. 2018;18(9):1513-1525.

26. Kato S, Murray J-C, Ganau M, Tan Y, Oshima Y, Tanaka S. Does posterior scoliosis correction improve respiratory function in adolescent idiopathic scoliosis? A systematic review and meta-analysis. Global Spine J. 2018;23(6):219256821881131.
Clinical Interventions in Aging

\section{Publish your work in this journal}

Clinical Interventions in Aging is an international, peer-reviewed journal focusing on evidence-based reports on the value or lack thereof of treatments intended to prevent or delay the onset of maladaptive correlates of aging in human beings. This journal is indexed on PubMed Central, MedLine,

\section{Dovepress}

CAS, Scopus and the Elsevier Bibliographic databases. The manuscript management system is completely online and includes a very quick and fair peer-review system, which is all easy to use. Visit http://www.dovepress. com/testimonials.php to read real quotes from published authors. 